\title{
Topical Treatment of Actinic Keratoses with Potassium Dobesilate 5\% Cream. A Preliminary Open-label Study
}

\author{
P. Cuevas ${ }^{1}$, W. Espinoza 2 , C. Pérez ${ }^{3}$, J. Angulo ${ }^{1}$, G. Giménez-Gallego ${ }^{4}$ \\ ${ }^{1}$ Departamento de Investigación. Servicio de Histología. IRYCIS. Hospital Universitario Ramón y Cajal, Madrid, \\ ${ }^{2}$ Servicio de Dermatología. Hospital Universitario Ramón y Cajal. Madrid, \\ ${ }^{3}$ Servicio de Farmacia. Hospital Universitario Ramón y Cajal. Madrid, \\ ${ }^{4}$ Departamento de Biología Fisico-Química, Centro de Investigaciones Biológicas Consejo Superior de Investigaciones Científicas (CSIC), \\ Madrid, Spain
}

\begin{abstract}
Background: Fibroblast growth factor (FGF) is involved in skin tumorigenesis: it promotes cell viability, induces angiogenesis and stimulates invasiveness. Dobesilate is a drug that blocks the activity of FGF. The primary objective was to evaluate the efficacy and tolerability of potassium dobesilate $5 \%$ cream in the treatment of actinic keratoses.

Methods: Potassium dobesilate 5\% cream was applied twice daily for 16 weeks to actinic keratosis lesions in 30 patients. The lesions were evaluated clinically at an initial baseline visit, at intermediate visits, and at 16 weeks of treatment.

Results: The use of potassium dobesilate 5\% cream for 16 weeks induced complete regression in $70 \%$ of evaluated actinic keratoses, corresponding to grade I, II and III clinical variants, and a partial response (at least $75 \%$ reduction of lesions) in $20 \%$ of the cases. Conclusion: Our preliminary trial shows that potassium dobesilate exerts anti-tumorigenic effects and may play a useful role in the chemoprevention of skin cancers.
\end{abstract}

Key words: Actinic keratosis; Dobesilate; Fibroblast growth factor.

\section{INTRODUCTION}

Actinic keratoses (AKS) are rough, scaly lesions that commonly occur on sun-exposed areas of the skin. AK represents the most common carcinoma in situ of the skin, showing a continuously increasing incidence worldwide[1]. AK has been previously referred to as preneoplastic lesions, but is nowadays defined as intraepidermal carcinoma progressing towards invasive squamous cell carcinoma (SCC) [2]. Histologically, AK lesions are characterized by an epidermal proliferation of atypical keratinocytes starting from the basal cell layer. According to the epidermal involvement of dysplasia, three subtypes of AK lesions may be differentiated: in situ SCC type AK I (mild), in situ SCC type AK II (moderate) and in situ SCC type AK III (severe) [3].

Until now, the decision to treat AK lesions was based on cosmetic reasons and to relieve symptoms. However, a general consensus currently exists to treat AK lesions for prevention of malignancy and metastasis. Treatment options include ablative (destructive) therapies such as cryosurgery, curettage with electrosurgery and photodynamic therapy, and topical therapies generally used in patients with multiple lesions.

For topical treatment, fluorouracil has been a traditional topical treatment for AK, although imiquimod $5 \%$ cream and diclofenac 3\% gel are effective alternative therapies. Fluorouracil is useful for treating multiple and subclinical lesions, but a long period is often required to treat deep and hyperkeratotic AK lesions. The drug can cause wound infections, ulcers and scarring, and has a reported clearance rate of 50\% [4]. Imiquimod $5 \%$ cream is effective in treating $\mathrm{AK}$, with a complete response reported in $45-75 \%$ of patients. However, during treatment, patients generally present with adverse events, ranging from redness to hemorrhagic crusted lesions [5]. $3 \%$ diclofenac in 2,5\% hyaluronic acid gel is a topical non-steroidal anti-inflammatory drug (NSAID) formulation that offers an effective approach to treat $\mathrm{AK}$, showing complete healing of AK lesions in 50\% of treated lesions [6]. Adverse effects associated with topical diclofenac were pruritus (25\% of patients), mild erythema (25\%), dry skin $(10 \%)$ and rash $(2 \%)[7]$.

On the basis of the successful therapeutic effect of dobesilate in preclinical models of cancer and in a case-control study of basal cell carcinoma (BCC) [8], we investigated - to our knowledge for the first time the use of potassium dobesilate $5 \%$ cream in the treatment of AK lesions. Dobesilate is a new well-characterized and efficient synthetic fibroblast growth factor (FGF) inhibitor[9] that has been used for many years as a vasculotropic agent without any known side effects.

\section{Methods}

This open-label trial was conducted to evaluate the efficacy and tolerability of potassium dobesilate 5\% cream in the treatment of patients affected by AKs. This was a study conducted at a single academic study center.

\section{PATIENTS AND TREATMENT}

We enrolled 46 patients ( 12 women and 34 men) from 64 to 90 years (mean age 76.9 years) with clinical diag- 
nosis of non-hyperkeratotic grades I (mild) and II (moderate) and hyperkeratotic grade III (severe) actinic keratoses. All patients signed a written informed consent before initiating treatment, and the study was conducted in accordance with the ethical guidelines of the Declaration of Helsinki.

All patients presented at least, one AK lesion localized on the face $(52 \%)$ or the scalp (48\%). 17.4\% of the patients presented grade I AK lesions, $43.5 \%$ grade II AK lesions and $39.1 \%$ presented the most severe variant, grade III AK lesions.

Potassium dobesilate 5\% cream was prepared at the Pharmacy Department of the Hospital Universitario Ramón y Cajal in Madrid, Spain. This formulation does not contain any keratolytic agent. Topical treatment was self-applied by the patient twice a day on AK lesions for 16 weeks.

\section{ASSESSMENT}

To assess the efficacy of the therapy, we clinically evaluated the lesions at an initial baseline visit, at intermediate visits, and after 16 weeks (endpoint). For the primary efficacy variable (complete clearance of lesions), patients were examined 8 weeks after the end of treatment. Cosmetic outcome was assessed by investigators and was based on the amount of scarring, atrophy, induration, erythema and pigmented change within the treated area in comparison with adjacent, untreated skin. It was graded as excellent (no erythema, change in pigmentation, scarring, atrophy or induration), good (slight to moderate erythema or change in pigmentation but no scarring, atrophy or induration), fair (slight scarring, atrophy or induration) or poor (moderate to extensive scarring, atrophy or induration).

\section{RESULTS}

Twelve patients were lost in follow-up and 4 patients violated protocol (did not apply cream for 4 weeks or more). Thirty patients completed the 16 weeks treatment period and 8-week post-treatment follow-up. After 16-week of treatment there was improvement in AK lesions. Indeed, potassium dobesilate 5\% cream induced a complete clearance in $21 / 30$ patients $(70 \%)$; $20 \%$ of patients $(6 / 30)$ had partial response (at least $75 \%$ reduction of lesions), and $10 \%$ of patients $(3 / 30)$ showed less than $75 \%$ or no clinical improvement. Of the 21 patients with complete clearance of lesions, the basal grade of AK was: grade I: 5 patients $(23.8 \%)$, grade II: 7 patients $(33.3 \%)$ and grade III: 9 patients $(42.9 \%)$. Of the 30 patients who completed the protocol, clearance according to basal grade of AK was: grade I: $100 \%$ (5/5); grade II: $63.6 \%(7 / 11)$ and grade III: $64.3 \%(9 / 14)$.

In the 30 patients who completed the protocol, the clinical response according to $\mathrm{AK}$ lesion localization was; a) face: $11 / 14$ patients $(78.6 \%)$ showed complete clearance and $3 / 14$ patients $(21.4 \%$ ) a partial clearance; b) scalp: $10 / 16$ patients $(62.5 \%)$ had a complete clearance; $4 / 16$ patients $(25 \%)$ a partial clearance and in $2 / 16$ patients $(12.5 \%)$ had no response. Five patients cleared their AK lesions before the end of treatment. Figure 1 shows that complete remission was ob- tained in AK lesions as early as at 15 days and 30 days of treatment.

In our sample, there was an excellent post-treatment cosmesis after dobesilate application. None of the patients abandoned treatment because of side effects nor did they report any systemic adverse effects related to the use of the topical formulation of dobesilate. In some patients who have completed the study, local reactions have been observed restricted to the AK lesion under treatment and were graded as mild, moderate or severe by the patient and physician. Local observed effects were pruritus: mild $(5 / 23,22 \%)$ and stinging: mild $(4 / 23,17 \%)$. One of the patients presented moderate erythema outside of the AK lesions at 4 weeks of treatment, an adverse reaction which completely disappeared at 8 weeks of treatment.

\section{Discussion}

In order to grow beyond minimal size and to metastasize, tumors need to induce the growth of new blood vessels (angiogenesis)[10]. Whereas in normal tissues, vascular quiescence is maintained by the dominant influence of endogenous angiogenesis inhibitors over angiogenic stimuli, tumor angiogenesis is induced by increased secretion of angiogenic factors and/or by downregulation of angiogenesis inhibitors. Because angiogenesis occurs in hypertrophic AK and SCC [11], antiangiogenic therapy could be an attractive approach to treat such malignancies.

Dobesilate has been shown to exert anti-tumorigenic effects through inhibition of FGF [8, 9, 10]. FGF participates in oncogenesis by stimulating cell proliferation, survival, invasion and angiogenesis. The crucial role of FGF in tumor development has been reported in animal studies in which abrogation of FGF signalling abolishes tumor establishment and progression [12]. Thus, inhibition of FGF production by tumor and endothelial cells could be a putative novel target for cancer therapy. Furthermore, FGF has been shown to be upregulated in skin cancers [13], and inhibition of FGF signaling in human melanoma cells abolished experimental melanoma growth in the rabbit avascular cornea [14]. In addition, in a case-control study, topical application of dobesilate showed clinical efficacy in BCC [9]. AK lesions start as localized clonal expansions of ultraviolet light-damaged keratinocytes. It is possible that local application of dobesilate acts by selectively destroying or reprogramming them to a more normal pattern of differentiation. This report supports the use of dobesilate as an antiangiogenic agent to treat actinic keratosis. Our data also suggest that dobesilate may also be used to treat a broad range of angiogenesis-dependent dermatological conditions, including squamous cell carcinoma (SCC) and basal cell carcinoma (BCC).

\section{CONCLUSIONS}

Actinic keratosis $(\mathrm{AK})$ is a squamous cell carcinoma (SCC) in situ, and its eradication is mandatory in affected patients. Our clinical experience shows a new potential application of potassium dobesilate 5\% cream in the topical treatment of AK lesions. Dobesi- 

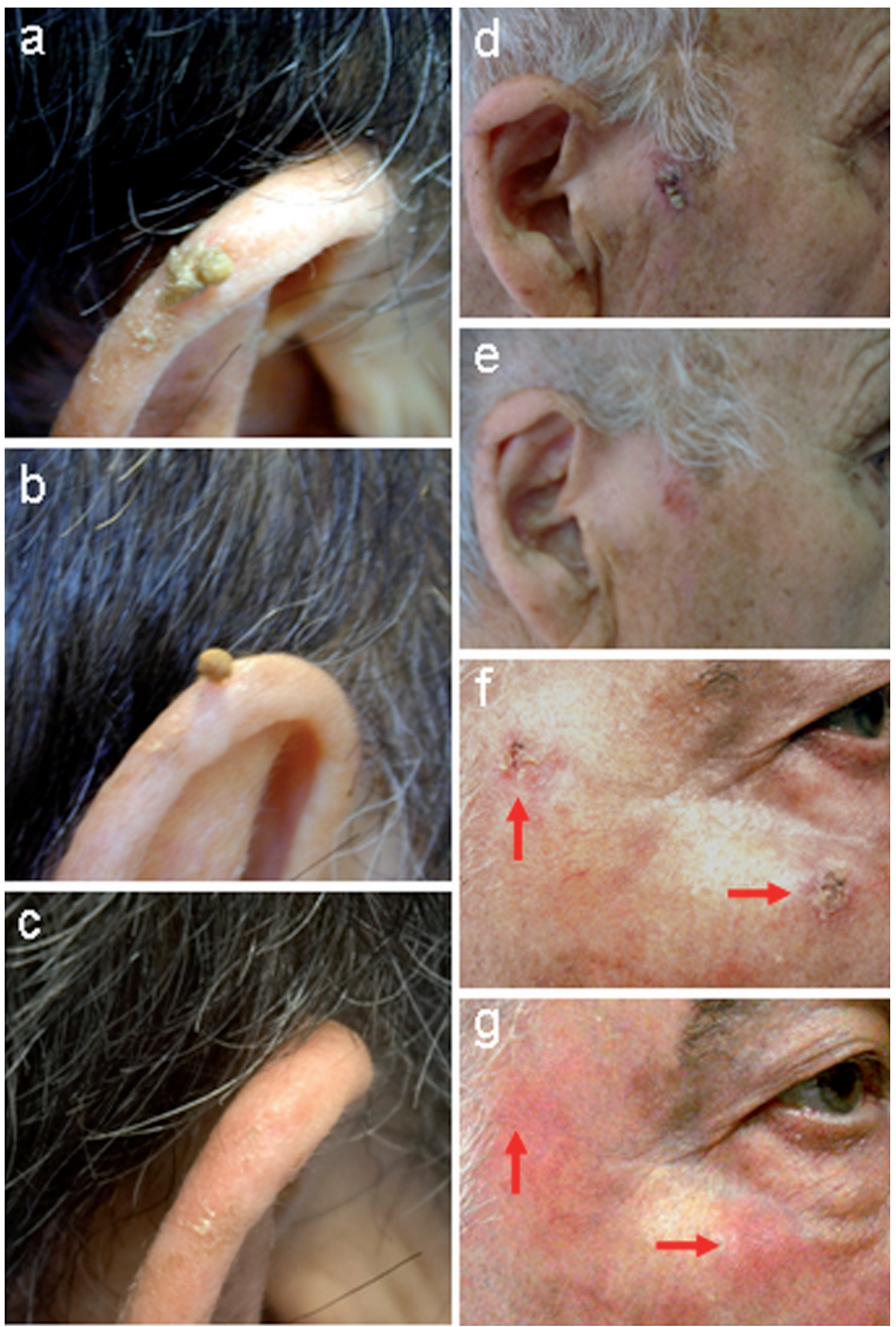

Fig. 1. Efficacy of dobesilate in hypertrophic actinic keratosis lesions. Keratotic actinic keratosis in three patients treated with potassium dobesilate 5\% cream twice a day. Left panels depict actinic keratosis on the ear of patient $\mathrm{N}^{\circ} 3$ before (a), at 12 days (b) and clinical remission of lesion after 15 days of treatment (c). Actinic keratotic lesions on the face of patient $\mathrm{N}^{\circ} 8$ (d,e) and patient $\mathrm{N}^{\circ} 9(\mathrm{f}, \mathrm{g})$ at baseline $(\mathrm{d}, \mathrm{f})$ and after 30 days of treatment $(\mathrm{e}, \mathrm{g})$. 
late appears to be effective, safe, and well tolerated. Larger placebo-controlled clinical trials are warranted to compare the effectiveness of this well known antiFGF compound in the treatment of AK lesions. However, our preliminary results suggest that potassium dobesilate $5 \%$ cream may play a key role in the chemoprevention of skin cancers, which could have a significant public health impact.

\section{Acknowledgements:}

This work was supported by CONSOLIDER CSD200900088 form the Spanish Ministry of Science and Innovation and by a grant from Action Medicines S.L:, Spain. We thank C. Bourdier for assistance in elaborating the manuscript.

\section{REFERENCES}

1. Selasche SJ. Epidemiology of actinic keratoses and squamous cell carcinoma. J Am Acad Dermatol. 2000; 42: 4-7.

2. Ackerman AB. Solar keratosis is squamous cell carcinoma. Arch Dermatol. 2003; 139: 1216-7.

3. Röwert-Huber J, Patel MJ, Forschner T et al.. Actinic keratosis is an early in situ squamous cell carcinoma: a proposal for reclassification. Br J Dermatol. 2007; 156 (Suppl 3): 8-12.

4. Gupta AK. The management of actinic keratoses in the United States wih topical fluorouracil: a pharmacoeconomic evaluation. Cutis. 2002; 70:30-6.

5. Lebwohl M, Dinehart S, Whiting D et al. Imiquimod 5\% cream for the treatment of actinic keratosis: results from two phase III, randomized double-blind, parallel group, vehicle-controlled trials. J Am Acad Dermatol. 2004; 50: 714-21.

6. Rivers JK, Arlette J, Shear N et al. Topical treatment of actinic keratoses with 3.0\% diclofenac in 2,5\% hyaluronic gel. Br J Dermatol 2002; 146:194-200.

7. Campione E, Diluvio L, Paterno EJ, Chimenti S. Topical treatment of actinic keratoses with piroxicam 1\% gel. A preliminary open-label study utilizing a new clinical score. Am J Clin Dermatol. 2010; 11: 45-50.
8. Cuevas P, Arrazola JM. Treatment of basal cell carcinoma with topical dobesilate. J Am Acad Dermatol. 2005; 53: 526-7.

9. Fernández IS, Cuevas P, Angulo J et al. Gentisic acid, a compound associated with plant defence and a metabolite of aspirin, heads a new class of in vivo FGF inhibitor. J Biol Chem. 2010; 285:11714-29.

10. Folkman J. Angiogenesis in cancer, vascular, rheumatoid and other disease. Nat Med. 1995; 1: 27-32.

11. Strieth S, Hartschuh W, Pilz L, Fusening NE. Angiogenic swith occurs late in squamous cell carcinomas of human skin. Br J Cancer. 2000; 82: 591-600.

12. Wang Y, Becker D. Antisense targeting of basic fibroblast growth factor receptor- 1 in human melanomas blocks intratumoral angiogenesis and tumor growth. Nat Med. 1997; 8: 887-93.

13. Arbiser JL, Byers HR, Cohen C, Arbeits J. Altered basic fibroblast growth factor expression in common epidermal neoplasms: examination with in situ hybridization and immunohistochemistry. J Am Acad Dermatol. 2000; 42: 973-7.

14. Cuevas P, De Miguel R, Espinoza W et al. Antimelanoma effect of a synthetic inhibitor of fibroblast growth factor (FGF) [abstract $\mathrm{n}^{\circ} 79$ plus Poster]. 13th World Congress on Cancers of the Skin; 2010 April 7-10. Madrid (Spain).

Received: August 20, 2010 / Accepted: October 20, 2010

Address for correspondence:

Pedro Cuevas Sánchez M.D. Ph.D.

Servicio de Histología

IRYCIS

Hospital Ramón y Cajal

Ctra. de Colmenar km. 9.100

28034-Madrid

Spain

Tel/Fax: (34)913368290

E-mail: pedro.cuevas@hrc.es 